\title{
Teaching integration with layers and representations: A case study
}

\author{
Joshua Von Korff and N. Sanjay Rebello \\ Department of Physics, Kansas State University, 116 Cardwell Hall, Manhattan, Kansas 66506-2601, USA
}

(Received 24 November 2011; published 18 May 2012)

\begin{abstract}
We designed a sequence of seven lessons to facilitate learning of integration in a physics context. We implemented this sequence with a single college sophomore, "Amber," who was concurrently enrolled in a first-semester calculus-based introductory physics course which covered topics in mechanics. We outline the philosophy underpinning these lessons, which characterizes integration in terms of layers and representations. We describe how Amber learned to give oral presentations in which she told a story about how integration comes from products, sums, and limits in a variety of physics contexts. We conclude that by the end of our lessons, Amber was able to conceptualize and explain integrals using multiple representations. In one case, she was able to solve a novel problem about integration in an unfamiliar context (center of mass.) Based on our previous research about integration, we suggest that these achievements would have been unattainable with the use of a single one or two hour lesson.
\end{abstract}

DOI: 10.1103/PhysRevSTPER.8.010125

PACS numbers: 01.40.Fk, 01.40.G-

\section{INTRODUCTION}

Physics students are often required to perform integrals in contexts ranging from mechanics (finding displacement using $\int v d t$ ) to introductory electricity and magnetism (finding the electric field produced by a line of charge using $\int k \frac{d q}{r^{2}}$ ) to thermodynamics (finding work using $\left.\int P d V\right)$. However, research by Meredith and Marrongelle has shown that students may rely on cues to decide when an integral is necessary, including recall, dependence (a quantity that varies with another quantity), and parts-of-a-whole cues [1]. When such cues are inadequate to decide how to solve a problem or answer a question, we might expect that students would not know what to do.

For example, Nguyen and Rebello asked students a question about resistance that required integrating a varying resistivity along a cylindrical conductor [2]. Most students said that an integral was needed "because the resistivity was changing along the conductor," making use of a "dependence" cue. However, when asked to interpret infinitesimal quantities such as $d x$ or $d A$, students were out of their depth, for instance saying that $d A$ refers to a changing area, when in fact it refers to a small element of area. Helping students to differentiate between a change $d A$ and an area element $d A$, and to use these infinitesimals correctly, may require some instruction targeted at the student's physical intuition about infinitesimals. It is this kind of understanding that we addressed in this research.

Although cues may be reliable under some circumstances, we would like to encourage students to build a more robust conceptual understanding that they can express in

Published by the American Physical Society under the terms of the Creative Commons Attribution 3.0 License. Further distribution of this work must maintain attribution to the author(s) and the published article's title, journal citation, and DOI. words or diagrams. We designed a sequence of seven lessons about integration in the context of introductory mechanics and observed the progress of one student, "Amber," as she participated in the lessons. Amber was a sophomore who had previously taken one semester of college calculus and was simultaneously enrolled in a second semester of calculus. This simultaneous calculus course covered simple two-dimensional integrals, for instance, with functions that are constant in one of the two dimensions, but the course did not discuss more general situations.

Because this work is a case study, involving a sequence of lessons with a single student, we are unable to provide any specific recommendations as to how our findings may be generalized. It may be that most students would approach our lessons as Amber did, or on the other hand, it may be that Amber's experience is unique. Further research would be required to address such questions. However, we hope that our case study can illustrate how a particular kind of instruction and assessment could be implemented and how at least some students might respond to such instruction. Individual instructors will have to decide for themselves whether their students would benefit from our lessons. We are hopeful that the ideas presented in this paper would be suitable for students with less preparation than Amber, or could be adapted for such students. Especially in the first few lessons, we did not require Amber to perform complex algebraic computations or set up a large number of equations, so it is possible that students with less skill at solving traditional physics problems could still excel in the context of these lessons. However, further work would be required to investigate this possibility. We believe that of the seven lessons, the last three required the greatest amount of physics preparation.

In this paper, we will first consider what it means to understand integration in a physics context. Toward this 
end, we will begin by outlining Zandieh's framework [3] on this topic, elaborating on the role of "layers" such as sums, products, and limits. Then we will describe our own framework for what it means to understand integration. Briefly, our view is that a student who can engage with integrals' structure using sums, products, and limits, and in several representations, such as spoken or written words, graphs, tables, equations, and diagrams, can be said to understand integration. All of these layers and representations are studied in the present work. Next, we will discuss how this perspective is related to the frameworks of Zandieh, Thompson and Silverman [4], and Sealey [5]. We will describe the content of the seven lessons we constructed to facilitate student understanding of integration, and discuss the motivation underlying our construction of these lessons. Finally, we will consider Amber's reaction to the lessons and draw some conclusions.

We will not discuss all aspects of students' understanding of integration. Notably, we have not asked Amber to think about limits of integration, even though all definite integrals have limits of integration. There is also a great deal to be said about the " $d$ " notation, used to indicate infinitesimals, and we hope to address this issue in greater depth in a future paper. Notational and symbolic aspects of the integral are a part of the equation representation in our framework.

\section{WHAT DOES IT MEAN TO “UNDERSTAND INTEGRATION"?}

In this section, we posit what we mean by understanding integration. To do so, we describe what we mean by two structures: (a) layers and (b) representations. We explain how these structures are important for understanding integration.

\section{A. Layers}

Several researchers have discussed the "layer" structure of differentiation and integration, for instance, the relationship between integrals and sums, or between derivatives and ratios. Zandieh describes a layered framework for students' understanding of derivatives [3] based on Sfard's process-object complementarity [6]. We will first explain Sfard's ideas, then discuss Zandieh's framework. Then, we will describe a similar framework for integration in a physics context.

Sfard's main idea is that mathematical entities can be conceived of as either processes or objects, and that a person must first possess a procedural conception, which later becomes a structural (object) conception. This natural order of learning follows the same path as the historical development of mathematics, which also turns processes into objects ("reification"). For instance, concrete objects were counted (process) before the idea of number (object) was invented to reify this process. Numbers were subtracted (process) before the idea of a negative number (object) was invented to reify this process.
According to Sfard, many previous authors have made similar distinctions, such as conceptual or procedural $[7,8]$, relational or instrumental [9], and others. Sfard's contribution is to see these as dualities rather than as dichotomies. A dichotomy is a pair of concepts that are mutually exclusive opposites. A duality is a pair of concepts that may seem to be opposites but are really two aspects of a single phenomenon. (Sfard uses the example of the particle-wave duality in quantum mechanics.)

Sfard concludes that reifying, say, negative numbers, requires starting to learn procedures that utilize them. [Like being able to solve $5-(-2)$.] That is, sometimes you have to learn the procedure without understanding the concept. But it may happen that students, having memorized the procedure, have no further motivation to understand the underlying object " -2 ," and never attain any deeper understanding at the conceptual level. In the context of calculus, taking a limit of the ratio $\lim _{h \rightarrow 0} \frac{f(x+h)-f(x)}{h}$ might be considered a process, where we imagine $h$ shrinking, but the result of this process is a single number, which is an object. Thus, the derivative is both a process and an object. Zandieh analyzes students' understanding of derivatives through the lens of Sfard's process-object duality, pointing out three layers of the student's understanding: the ratio, limit, and function layers. Each layer can be viewed as a process or an object. We briefly describe each layer:

Z1. Ratio layer-The most basic concept in the construction of a derivative is the idea of a ratio. Students should understand the connection between formulas such as $\frac{f(x+\Delta x)-f(x)}{\Delta x}$, the procedure for finding the slope of a graph by dividing "rise over run," and the idea of average velocity as the ratio $\frac{\Delta x}{\Delta t}$.

Z2. Limit layer-Next, the student should be able to imagine the denominator of the ratio approaching zero. In the graphical case, this means visualizing the two points on the curve getting closer and closer together. In the kinematics representation, the student should imagine a particle moving over a short displacement during a short time interval, as the length of that interval becomes less and less.

Z3. Function layer-Finally, the student is to conceive of the derivative as a function. That is, the derivative of the function $x^{3}$ is $3 x^{2}$, which is a statement about one function $\left(x^{3}\right)$ becoming another function $\left(3 x^{2}\right)$. The student could understand this by visualizing a tangent line at every point along a graph, instead of at just one point.

Each layer can be conceived as either a process or an object, and each process-object pair can be represented in the form of an equation, graph, verbalization, or kinematic motion. For instance, the slope of a line can be determined by the process of measuring a rise and a run, and dividing one by the other. Once the student understands this process well enough to perform operations on the slope itself (such as the operation of imagining a limit of secant slopes rotating to become a tangent line), the student is said to understand the slope as an object. 
This framework gives no account of procedural rules for taking derivatives of specific functions. For instance, the derivative of $f(x) g(x)$ is $f^{\prime}(x) g(x)+f(x) g^{\prime}(x)$. The student can utilize this rule without really understanding the derivative in Zandieh's sense. Sfard says that such a student possesses a "pseudostructural" understanding; the student understands that there is an object called "derivative," and is able to perform operations with it, but is unable to explain its internal structure, such as the ratio layer that is used to define the derivative. In general, a student's understanding is said to be pseudostructural if they do not understand the layers that would be used to define that object. For instance, a student who can integrate by parts, but does not understand that the integral is a sum, would be said to have a pseudostructural understanding of the integral. Another way of saying that a student has "pseudostructural" understanding is to say that they comprehend the derivative as a "pseudo-object" (Zandieh's term, [3]). A "pseudo-object" is any object that the student understands in a pseudostructural way.

The concept of integration can be dissected in a similar way to Zandieh's method. Our approach is very close to Thompson and Silverman's accumulation of "multiplicative bits" [4], as well as Sealey's layered framework [5]. We will first describe our method, then compare it with the frameworks of Zandieh, Thompson and Silverman, and Sealey. Our framework describes integration using a network of processes and objects, with multiple routes to understanding. Although the network as a whole is complex, we are not asserting that successful instruction must comprehend all possible routes.

Figure 1 depicts a network of eight objects, numbered VR1-4 and VR1-4*. There are also 10 arrows, which represent the processes that transform or construct these objects. Our instruction focuses on building the integral, (VR3*), but the sum and integral functions (VR4) and (VR4*) are included for the sake of completeness. By the "sum function" or "integral function," we mean the sum or integral considered as a mathematical function of its upper limit of integration (or upper limit of summation). As the upper limit of integration varies, the value of the sum or integral will vary; therefore, the sum or integral can be viewed as a function of that limit. See sections (VR4) and (VR4*) below for further explanation.

Macroscopic quantity (VRI) and infinitesimal quantity $(V R I *)$.- In most physical integrals, a variable of integration is either a change (as $d t$ or $d x$ represent a change in time or position) or an amount (as $d m$ represents an amount of mass). When the changes or amounts are macroscopic, they may be represented with a " $\Delta$ " (change in time $\Delta t$ ) or with no symbol at all (mass $M$ ).

Macroscopic product (VR2) and infinitesimal product $(V R 2 *)$. - The product layers represent the integral of a constant function, which may be represented in several ways. The function could be written out formally, or it could be described in words, or depicted as a rectangle on a graph. In a physics context, it could take the form of a particle moving at constant velocity or a rod of uniform density. In any of these representations, the integral of the constant function can be found by multiplication. This multiplication process constructs an "area" or "product" object, so this layer could be called the "product layer." One can construct the "infinitesimal product layer" by shrinking the rectangle to have a small width; in other words, $v \Delta t$ becomes $v d t$.

Sum (VR3) and integral (VR3*).-Next, one has to approximate the integral of a general function by a piecewise-constant (step) function, whose integral is a sum of products. On a graph, the step function looks like a staircase. To compute the approximate integral, one must sum the areas of the rectangles, or sum the products $f(x) \Delta x, v \Delta t$, etc. In this case, an approximation process constructs a sum object. This layer could be called the "summation layer." In equation form, the sum might look like $\sum_{i=0}^{n} v(i \Delta t) \Delta t$. When the widths of the rectangles approach zero, the sum becomes an integral. In our framework, the integral can also be constructed by summing an "infinite" number of infinitesimal products (VR2*), although a traditional calculus framework would not allow this.

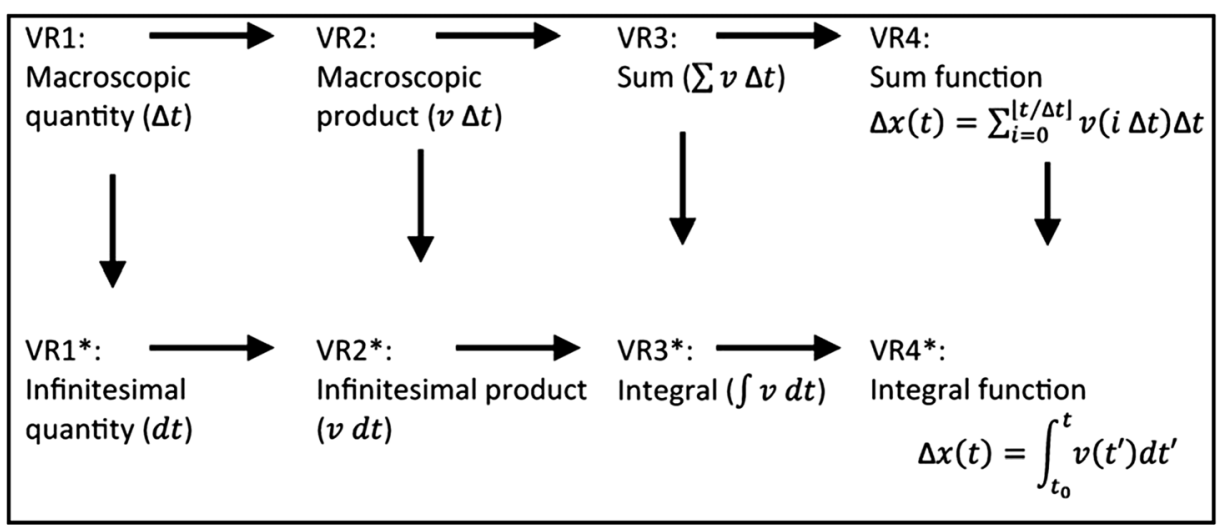

FIG. 1. A network of process-object routes to understanding integration. 
Sum function (VR4) and Integral function (VR4*).-By varying the number of terms " $n$ " in the macroscopic sum, while keeping the interval width $\Delta x$ constant, we obtain a function. This function could be written as

$$
F(x)=\sum_{i=0}^{\left\lfloor\frac{x}{\Delta x}\right\rfloor} f(i \Delta x) \Delta x,
$$

following Thompson and Silverman [4]. When the interval $\Delta x$ approaches zero, we imagine a continuous function. That is, an integral such as $\int_{0}^{x} x^{\prime} d x^{\prime}=\frac{x^{2}}{2}$ could be conceptualized as a function of $x$ allowing many possible inputs, $g(x)=\int_{0}^{x} x^{\prime} d x^{\prime}=\frac{x^{2}}{2}$, rather than just a statement about a particular value of $x$. This is analogous to Zandieh's final layer, in which the derivative value becomes a derivative function.

Table I depicts all eight of our layers and the corresponding layers in other frameworks. Sealey includes a "prelayer" which she calls "orienting." This has to do with sense making. For instance, in one of Sealey's problems, students used integration to find the force exerted on the surface of a dam, assumed to be a rectangular vertical wall, where the pressure is proportional to the depth. In this context, the student must integrate $P d A$. Amber worked on a similar problem in our seventh and last lesson. Sealey said that when solving this problem, "Until the students were able to begin making sense of the meanings of pressure and force, they did not see a need for approximations “ [5]. Because Sealey's prelayer involves understanding the meanings of physical quantities, it is related to our "macroscopic quantity" layer.

Our framework differs from Zandieh's [3] primarily in that we have analyzed integration, whereas she has analyzed differentiation. Our framework is unlike all three frameworks-Zandieh's, Thompson and Silverman's [4], and Sealey's [5] - in that we encouraged Amber to understand infinitesimal quantities (VR1*) and infinitesimal products $(\mathrm{VR} 2 *)$ on their own terms. Zandieh considers ratios of finite quantities, and limits of those ratios, but not ratios of infinitesimal quantities. While Thompson and Silverman briefly mention the role of infinitesimals, they focus on the more traditional approach involving Riemann sums. According to the mathematical framework taught in a standard calculus class, it is useless to take the limit of an infinitesimal such as " $v d t$ " prior to summation, since this limit would be zero. Instead, one should take the limit of a sum of finite quantities, as the number of those quantities approaches infinity. But from a physical point of view, students can be taught that an integral is "the sum of many infinitesimally small quantities," without harm to their understanding. Therefore, our framework allows for multiple process-object routes to understanding integration, as depicted in Fig. 1.

\section{B. Representations}

Several authors have considered representations in the context of integration. Sealey [5] considers algebraic, numerical, graphical, and contextual (related to the physical context) representations. Ghazali et al. [10] measured students' understanding by asking them to translate integral concepts between symbolic, graphical, and verbal representations. Ghazali's definition of student understanding of integrals included four constructs: basic definitions, basic operations, applications, and translation between representations.

In a mathematical context, the most important representations may involve graphs and equations, but in a physics context, a physical representation is of paramount importance. That is, we want students to understand what they are adding up and why, not only that they are adding something up. Physics students must learn to work with verbal representations, as well as diagrams, concrete physical objects, and other representations that connect with their informal knowledge about how the world is put together.

When integrating velocity to find displacement, the graphical representation involves plotting a function $v(t)$, dividing the picture into columns, and imagining the column widths shrinking to zero. But one might ask: why are we taking an integral at all? And why are we taking this integral? True, the equation $x=v t$ cues us that an integral might be appropriate, by suggesting the product layer: the area of a rectangle could be found by multiplying $v t$. And the idea of "varying velocity" is another cue. But even in

TABLE I. We list all eight of our layers, with corresponding equations. VR indicates Von Korff and Rebello, S indicates Sealey, and TS refers to Thompson and Silverman.

\begin{tabular}{lll}
\hline \hline Layer number & \multicolumn{1}{c}{ Layer name } & Equation representation \\
\hline VR1, SPre & VR: Macroscopic quantity; S: Orienting & $\Delta t$ \\
VR1* & Infinitesimal quantity & $d t$ \\
TS1, VR2, S1 & Macroscopic product & $\Delta x=v \Delta t$ \\
VR2 $*$ & Infinitesimal product & $d x=v d t$ \\
TS2, VR3, S2 & Sum & $\Delta x=\sum v_{i} \Delta t_{i}$ \\
VR3 ${ }^{*}, \mathrm{~S} 3$ & Integral & $\Delta x=\int v d t$ \\
TS3, VR4 & Step function & $\Delta x(t)=\sum_{i=0}^{\lfloor t / \Delta t\rfloor} v(i \Delta t) \Delta t$ \\
TS4, VR4*, S4 & Integral function & $\Delta x(t)=\int_{t_{0}}^{t} v\left(t^{\prime}\right) d t^{\prime}$ \\
\hline \hline
\end{tabular}


that circumstance, not all products in physics should be turned into integrals in the same way. For instance, the center of mass for two particles can be found by the expression $\frac{m_{1} x_{1}+m_{2} x_{2}}{m_{1}+m_{2}}$, but even in the continuous case the numerator's products " $m x$ " do not turn into $\int m(x) d x$. [It is not even clear what $\int m(x) d x$ would mean.] And even assuming that we should make an integral out of $x=v t$, why are we plotting $v(t)$ and not $t(v)$ ?

These questions cannot be answered using the graphical or equation representations alone. We can answer them only with reference to a mental model of integration, which implies not only a graphical or formulaic conception of the integral, but also a diagrammatic or simulative understanding of the physical system, one which incorporates the physical meanings of the variables. When we use the term "mental model," we refer specifically to the definition by Johnson-Laird [11], who defines mental models as "structural analogues of the world."

We hoped that Amber would become familiar with diagrams and mental simulations that are structurally similar to the physical phenomena. Graphs and equations are important, but these representations do not carry information about the geometric meanings of the physical quantities. Our intervention also introduced concrete physical objects, such as carts and springs, in order to help Amber to make the connection between representations and the objects they describe.

\section{CONSTRUCTIVISM AS A PEDAGOGICAL STRATEGY FOR TEACHING INTEGRATION}

In our lessons, we hoped to facilitate Amber to construct an understanding of all representations and the connections between them. We wanted Amber to come to feel comfortable with all layers and representations of integration and to demonstrate her understanding by means of verbal presentations. However, because of the complexity of our subject matter, we chose to begin by discussing a limited set of layers and representations and to facilitate Amber's knowledge construction from this foundation. We pursued a constructivist strategy, beginning with ideas that would be familiar to Amber.

The word "constructivism" is used to describe a wide range of philosophies about the nature of learning and knowing [12]. According to our constructivist perspective on learning, a lesson should give students the opportunity to construct scientific ideas for themselves, starting from students' current ways of thinking. However, the lesson designer must consider which of the students' many ideas are most suitable as a basis for learning. In a lesson about integration, it might seem natural to begin with students' preexisting knowledge about equations, which they have studied in calculus classes. However, we chose to address Amber's calculus knowledge as little as possible. We were concerned that Amber's understanding from calculus might be largely pseudostructural; in other words, she might treat the integral as an unanalyzable conceptual unit, a pseudo-object [3]. This concern emerged from interviews we performed in a previous study [13], in which we guided students to view $\Delta x=\int v d t$ as an accumulation of small copies of the simpler equation $x=v t$. Many students in this previous study demonstrated pseudostructural knowledge, accepting $\Delta x=\int v d t$ as a rule that needs no explanation. We assume that a student has pseudostructural knowledge of integration if he or she does not use sums or products to interpret an integral, indicating that he or she thinks about the "integral" object without understanding the processes that have constructed it. For instance, one student said, "My Calc 1 teacher in high school, he was real big on like distance, velocity, area, always know how they relate ... he pounded that into our heads." But when this same student was unable to recall the integral of acceleration, he did not know any way to figure out the answer. At least one student was actively reluctant to consider a layered approach to integration even after we led him to understand it. When asked to explain why the displacement is the integral of the velocity, he acknowledged the legitimacy of the layered approach, but said, "Personally I tend to believe things that are told to me in an equation."

Rather than discussing calculus, our first lesson relied on Amber's prior knowledge about the motion of a car on a highway to help her arrive at an experiential understanding of the infinitesimal relationship " $d x=v d t$." By "experiential," we mean that Amber incorporated the language of ordinary experience, relating the car and its speedometer to physical quantities. Amber demonstrated her knowledge by solving a complicated debate problem (see the Appendix), which required her to say which of several hypothetical students were making correct statements. Once Amber made progress with this verbal understanding, we assigned symbols to the words, helping her to write down " $d x=v d t$ " as a symbolic expression of her idea. By the time the first lesson ended, we still had not yet discussed calculus. So we view our approach as constructivist, but we took care to build initially on Amber's physical intuitions rather than her calculus knowledge.

\section{METHODOLOGY}

Our participant Amber, who was concurrently enrolled in first-semester calculus-based physics, was paid to participate in this study. She did not receive course credit for her participation. Her small summer course, attended by only 10 students, required students to attend four lectures each week and was designed to cover a semester's worth of introductory mechanics material in two months. Of the 10 enrolled students, three volunteered for our research project after we announced the opportunity during their lab: Amber, Carmen, and Matt. However, Carmen and Matt chose not to participate after the first lesson. We suspect 
that conflicts with students' summer employment may have been a factor in reducing participation.

Amber had taken Advanced Placement Physics B in high school in the past, giving her a stronger than usual background relative to students who typically take our introductory mechanics class. ${ }^{1}$ Her math background was similar to her classmates'. Her introductory mechanics course contained little or no material about integration. Near the end of the semester, she felt that her strengths in the class were "solving mathematical equations and trig applications" and her weaknesses were "knowing when to apply certain formulas." Below, we enumerate the seven lessons and discuss the goals and plan for each. Lessons were two hours in duration and were taught by a single instructor. All sessions took place in the same room in which Amber and other students worked on physics labs for their mechanics course.

During the lessons, we sometimes provided Amber with materials that she could work on with minimal assistance. However, at other times the instructor participated in extended Socratic dialogues with Amber. When preparing Amber to give a presentation in lessons four and five, the instructor assisted her using cues and Socratic questions but avoided writing or drawing anything for her. The instructor delivered a long lecture (one hour) only once, in lesson three.

For most activities, Amber and the instructor were seated at the same table, discussing a worksheet or the lab equipment. These scenes could be videotaped by a stationary camera with no operator. When Amber presented at the blackboard, the instructor operated the camera, sometimes allowing the camera to zoom in on her writing. Video data proved to be crucial, because we came to feel that Amber's use of multiple representations illustrated her understanding of integration. By analyzing Amber's presentations on the blackboard, we gained a sense of the diversity and frequency of her links between representations. To count these links, we transcribed and coded the video data, noting events where Amber describes the same quantity, product, or integral using two different representations.

\section{SEQUENCE OF LESSONS}

In this section, we will describe the content of the seven lessons and analyze excerpts of discussion and presentations from several lessons. An asterisk (*) indicates that a problem assigned for this lesson can be found in the Appendix. Our lessons focused on one-dimensional integrals related to Amber's introductory mechanics course, including integrals for displacement, work, momentum, moment of inertia, and center of mass. In the final lesson, Amber worked with a simple two-dimensional integral, in which she integrated

\footnotetext{
${ }^{1}$ In the United States, "AP" or "advanced placement" refers to a challenging course for high school students. AP Physics B is an advanced high school physics curriculum which is intended to be at the same level as a college algebra-based physics course.
}

pressure over an area, although she solved this integral using only a single variable of integration.

\section{A. Lesson One}

Throughout lesson one, Amber was working with two other students, Carmen and Matt, who did not participate in later lessons.

1.a.* Amber and the other students discussed an extended "debate" or "conflicting contentions" problem $[2,14]$ about the motion of a car. According to the problem statement, a car's speedometer was untrustworthy, and three fictitious students were proposing physical quantities that might be helpful in measuring the car's speed. For instance, one fictitious student thought that the change in position since the beginning of the trip was relevant, whereas another suggested taking a small change in position, such as a mile. In all, nine different quantities were put forward: $x, v, t, d x, d v, d t, \Delta x, \Delta v$, and $\Delta t$. These quantities were described in words, and were not yet given the symbols $d x, d t$, and so forth, because we were concerned that students have a pseudostructural understanding of " $d x$ " and " $d t$. ." They have heard of " $d x$ " in calculus classes, but they may be sufficiently comfortable with it that they will resist thinking about what it "means." In the problem statement, a fictitious student, Claire, suggested that "a small change in velocity," such as $61 \mathrm{mph}-$ $60 \mathrm{mph}=1 \mathrm{mph}$, may be relevant to the computation. Carmen and Amber discussed this possibility:

Carmen: I think she's also right with the velocity, because like a small change would make a pretty big difference. So if she takes it at two different points, I mean I think it would reduce the amount of error.

Amber. Yeah. The only thing is, is that she's ... she's saying that $\mathrm{v}$ has to equal the change, cause if you plugged in one mile per hour, it would be, the equation (Amber is referring to the equation $x=v t$ ) would not work.

Carmen: Yeah. That would not work at all.

In this exchange, Carmen proposes an interpretation of the phrase "small change in velocity," suggesting that it has to do with reducing the amount of error. Amber, however, directs the group's attention to the numerical quantity, $1 \mathrm{mph}$, suggested by Claire as an example of a "change." Neither has used the word "subtract" or a synonym, so from this interaction we cannot discern whether Amber or Carmen has made a connection with the idea of subtraction. Clearly, the idea of a "change in velocity" is not trivial and merits some sense-making conversation. To simply assume students understand the notation " $d v$ " would have been premature.

After a long discussion, Amber and the other students concluded that a small change in position should be divided by a small change in time, and this would result 
in the velocity at a particular point in time. After they resolved this puzzle, we gave names to physical quantities such as $d x$ and $d t$. Therefore, Amber was able to connect the "macroscopic equation" $x=v t$ with the "infinitesimal equation" $d x=v d t$. The macroscopic equation is applicable only when $v$ is constant, or when $x$ and $t$ are small. The infinitesimal equation specifies that $x$ and $t$ are the quantities which can be made small in this case. We feel that it is the student's responsibility to learn how to transform the macroscopic equation into an infinitesimal equation using their understanding of physics, rather than by memorizing which infinitesimals correspond to which macroscopic equations. To deduce an infinitesimal equation, a student must use a mental model of the physical situation to comprehend why $d x=v d t$ is appropriate, and not, say, $d x=d v d t$ or $d x=t d v$. At the end of this discussion, we presented a mini lecture emphasizing that in physics $d x=v d t$ is just as valid as $v=d x / d t$. We addressed the product layer but not the integration layer, as we did not yet say that the $d x$ 's should be added up.

1.b. We presented Amber, Carmen, and Matt with a tabular representation depicting the velocity versus time for a cart. The students were asked to move a physical cart in a way that matched the numbers given in the table. They also filled in the position values on the table, by summing $\Delta x=\sum v_{i} d t_{i}$. While they were able to perform the summation procedure, constructing the equation with sigma notation and indices proved to be too difficult for them. We explained the sigma notation in a mini lecture.

\section{B. Lesson Two}

The Appendix contains several problems from lesson 2. One of them depicts a "sequence diagram," which is our term for a table depicting the physical variables associated with a moving object. (The table in lesson 1.b also took the form of a sequence diagram.) We speculate that this representation might help students to associate the table representation with motion, but we have no evidence for or

TABLE II. Connections Amber made between representations. A " 1 " indicates that Amber made this connection once during her presentation. "N/A" indicates that this cell is not applicable, either because the cell would indicate an invalid connection between a representation and itself, or because this same connection appears in another cell in this table, and the information is given in the other cell.

\begin{tabular}{|c|c|c|c|c|}
\hline & $\begin{array}{c}\text { Equation } \\
\text { or symbolic }\end{array}$ & Graph & $\begin{array}{c}\text { Experiential } \\
\text { (verbal) }\end{array}$ & Table \\
\hline Diagram & 0 & 0 & 1 & 0 \\
\hline $\begin{array}{l}\text { Equation } \\
\text { or symbolic }\end{array}$ & N/A & 1 & 1 & 1 \\
\hline Graph & N/A & N/A & 1 & 0 \\
\hline $\begin{array}{l}\text { Experiential } \\
\text { (verbal) }\end{array}$ & N/A & N/A & N/A & 1 \\
\hline
\end{tabular}

TABLE III. Connections Amber made between representations. A " 2 " indicates that Amber made this connection twice during her presentation. "N/A" indicates that this cell is not applicable, as discussed in the caption for Table II.

\begin{tabular}{|c|c|c|c|c|}
\hline & $\begin{array}{c}\text { Equation } \\
\text { or symbolic }\end{array}$ & Graph & $\begin{array}{c}\text { Experiential } \\
\text { (verbal) }\end{array}$ & Table \\
\hline Diagram & 4 & 0 & 2 & 0 \\
\hline $\begin{array}{l}\text { Equation } \\
\text { or symbolic }\end{array}$ & N/A & 0 & 2 & 0 \\
\hline Graph & N/A & N/A & 0 & 0 \\
\hline $\begin{array}{l}\text { Experiential } \\
\text { (verbal) }\end{array}$ & N/A & N/A & N/A & 0 \\
\hline
\end{tabular}

against this possibility. Note that in Tables II and III, the "diagram" row refers to an ordinary picturelike diagram, not to a sequence diagram.

2.a.* This problem gave a table of velocity values for the two-dimensional motion of a car and required Amber to sum $v_{x} d t$ and $v_{y} d t$ to fill in the $x$ and $y$ values in the table. She then drew a picture of the motion and wrote an equation that could be used to approximate $\Delta x$ and $\Delta y$.

2.b.* This debate problem presented several peoples' arguments about the correct equation for $\Delta x$ for the car's motion in (2.a). Amber was asked to explain which arguments are correct.

2.c. Amber constructed a graph that has an area given by the equation $\Delta x=v_{1, x} d t_{1}+v_{2, x} d t_{2}+\cdots$ that had been developed in (2.b). She considered what happens to the area if the time interval shrinks.

We had deliberately delayed asking Amber to associate the sum with the graphical representation until this point, because we believe that most of our students understand this transition in terms of pseudo-objects. That is, they remember that integrals have to do with sums and areas, but do not recall why, and do not associate these layers with the lower-level product layer.

2.d.* Amber was asked to tell a story (in writing) describing our progress from the macroscopic equation $x=$ $v t$ to the area under a curve.

This was her first chance to summarize the layers of integration, although we had not taught her about layers explicitly. She gave a correct explanation, but mentioned only a few representations. Amber did not discuss the infinitesimal equation $d x=v d t$, mentioning only the macroscopic equation $x=v t$. She recognized the importance of the graph, that the area of each rectangle was of the form $v t$, and that these quantities could be added up to find the displacement. She also knew that the limit should be taken as the time interval of the rectangle $d t$ went to zero. However, she did not draw a graph, table, or diagram of the situation. In terms of our layers framework, Amber connected (VR1 $\rightarrow$ VR2 $\rightarrow$ VR3 $\rightarrow$ VR3 ${ }^{*}$ ); that is, she talked about a product and then a sum before taking a limit. In the following, we have inserted "VR" to indicate Amber's layer transitions in terms of our framework: 
Amber: “... velocity $\times$ time elapsed can be represented by the area of a rectangle (VR1 $\rightarrow$ VR2), and each rectangle can be added up to calculate a total displacement $(\mathrm{VR} 2 \rightarrow \mathrm{VR} 3)$. Then show that the estimation gets more and more accurate as the interval of time is shortened, eventually to the theoretical value of $d t\left(\mathrm{VR} 3 \rightarrow \mathrm{VR} 3^{*}\right)$."

Storytelling and presentation are an important part of assessing and building a student's understanding. We believe it is possible for the student to comprehend the transition from each layer to the next, but to resist understanding the process holistically. The storytelling process requires the student to make those connections.

2.e. This problem addressed the connection between a concrete physical representation of motion and a graphical representation. For this purpose, we used McDermott's tutorial on "representations of motion" [15].

\section{Lesson Three}

3.a.* Amber observed a physical system (a cart on a track, attached to a string and weight threaded over a pulley) and listed any physical quantities, such as kinetic energy or displacement, that might be relevant to this situation. Then she performed the experiment, dropping the weight, and plotted quantities in a table. The purpose of this exercise was to make her think about extracting a list of physical quantities from a situation, which is a useful skill for setting up integrals in the context of a physics problem (see lesson 5.c). Amber had previously performed experiments with this equipment as part of her mechanics course, but we encouraged her to identify variables that may not have been required by her lab. We also asked Amber a few questions about infinitesimals, to remind her about what she had learned so far.

3.b. At this point, Amber was given a substantial lecture for the first time. Although lecture is often said to be an inefficient way of transmitting information, Bransford and Schwartz say that students can learn from a lecture if they are prepared by learning about "contrasting cases" that help them distinguish between the relevant concepts [16]. Our previous work helped Amber to distinguish between $d x$ and $x$. In the lecture, we discussed explicitly the difference between instantaneous or "point" quantities such as $v, x$, and $t$ (defined at a particular instant or point in time) and "change" quantities such as $d v, d x$, and $d t$ (defined as the difference between point quantities over an interval.) Amber's experiment with the cart, in lesson 3.a, provided the physical context for the lecture. The lecture was not entirely one-sided, and included some discussion with the student. Amber stated later that this lecture helped her to consolidate her understanding of the concepts, although we have no evidence of the lecture's efficacy beyond her assertion.

\section{Lesson Four}

4.a.* Amber gave a presentation about the layers of integration for the proto-equation $p=F t$, in the context of a person colliding with an airbag. "Proto-equation" is our term; it indicates that this equation, like " $x=v t$," is written in a deliberately vague form, requiring the student to decide whether " $p$ " should refer to an absolute momentum, a change in momentum, or a very small change in momentum. Amber prepared for the presentation with our assistance, as we helped her resolve any questions she had about the problem. She was often uncertain about how the collision would behave as a physical system or how to translate this behavior into a mental model. For instance, in the passage below, Amber suggests that the force should be constant over the course of the collision, but that an integrand "has to be like a curve," so she does not see how an integral is necessary in this situation:

Amber: Can you give me a quick reminder?

Researcher: What do you want to be reminded of?

Amber: Just like describing the integral.

Researcher: Yes.

Amber: Cause it's like force times a small change in time. Researcher: Okay.

Amber: But ... force is supposed to differ? I don't know. Cause it's like, it has to be like a curve.

Researcher: Okay. Right.

Amber: And I don't see how F changes ...

After some time:

Amber: I mean like during the impact, the force is gonna be the same, I should just multiply it by how long the impact lasts.

It seems as though Amber cannot decide whether the force from the airbag would change. These difficulties show that skill with integration cannot be judged separately from a student's understanding of the relevant physics. The very first layer, the "macroscopic quantity" layer (VR1), often proves to be the most challenging-what physical quantities are relevant in a problem and how do they behave? We also helped Amber by specifying a list of representations for her to use in the presentation. Amber's presentation lasted for seven minutes, during which we interrupted only minimally and without giving substantive advice. Figure 2 shows a sketch of the blackboard after Amber's lesson 4 presentation.

In her presentation, Amber correctly made connections between several representations. The pairs of representations are shown in Table II.

For instance, Amber relates experiential language with a force versus time graph by saying, "... the force on the airbag is gonna be slowly increasing. And then once the impact ends it's gonna start to decrease."

We counted Amber's statements as "connections" only if we judged them to be physically correct. In addition, if Amber related the same ideas multiple times using the 


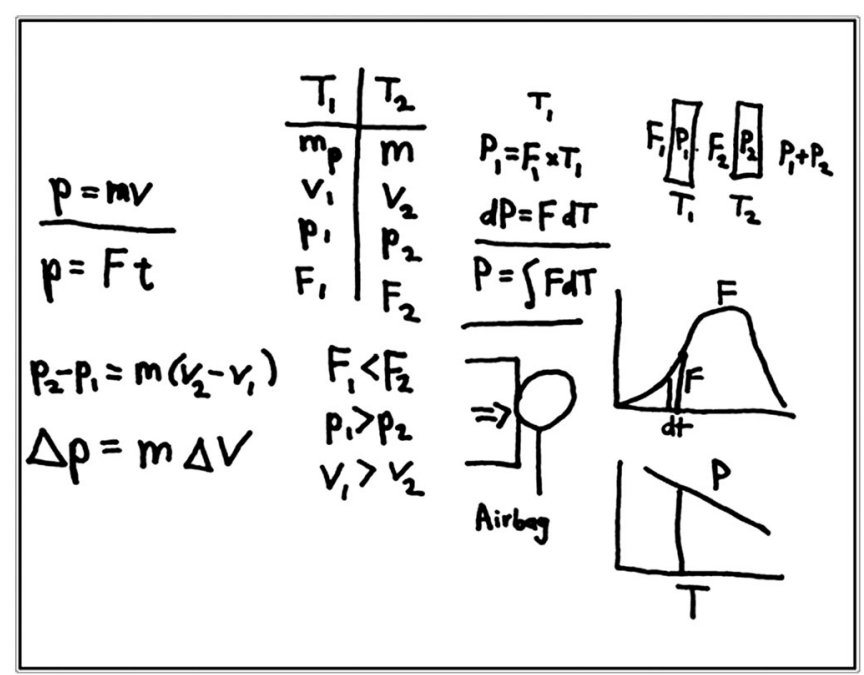

FIG. 2. Sketch of the blackboard after Amber's lesson 4 presentation. (Our videotaped screen shot would be difficult to read.) Amber uses both " $T$ " and " $t$ " to represent time.

same representations, we counted only a single connection. A discussion about an equation such as $d F=p d t$ was counted as one connection, even though the equation contains multiple physical quantities.

Table II does not capture the structure of layers that Amber discussed, nor does it show the way in which she connected clusters of more than two ideas. For instance, in this lesson, Amber connected the infinitesimal quantity (VR1*), product $(\mathrm{VR} 2 *)$, and integral (VR3*) layers. The excerpt below is annotated with (VR1*), (VR2*), and (VR3*) accordingly. Sometimes Amber mentions a process that can connect two objects on different layers. For instance, quantities can be multiplied to obtain a product, and products can be summed to obtain an integral. To illustrate these processes, we indicate the two layers and connect them by an arrow, like (VR1 $\rightarrow$ VR2 $\left.{ }^{*}\right)$.

Amber: For the, so an infinitesimal quantity, we will be looking at it in terms of, so, small change so that'd be $d p$ (VR2*) equal to $F$ times $\left(\mathrm{VR} 1^{*} \rightarrow \mathrm{VR} 2^{*}\right)$ really small change in time. (VR1*) [Writes: " $d P=F \cdot d T "$ "] So you are looking at it like this, so if you make a diagram [draws axes], and then, so the force on the airbag is gonna be slowly increasing. And then once the impact ends it's gonna start to decrease. [Draws (unlabeled) force vs time plot, with force increasing then decreasing.] So to find this really small change in time (VR1*), you will still be taking the areas (VR2*) except you are just gonna have to[Draws a thin column rectangle on the force vs time plot] (VR2*) so hypothetically to find the most exact, this is gonna be a very small change in time (VR1*) [indicates bottom of column], and this is still gonna be equal to the force [indicates top of column]. So you take $d t$ (VR1*) times
$\left(\mathrm{VR} 1^{*} \rightarrow \mathrm{VR} 2^{*}\right)$ whatever the height is at this point. So to add up all those quantities (VR2* $\rightarrow$ VR3*) you'd end up with the equation [Writes" $P=$ "], so the integral (VR3*) is taking the sum of all of these little quantities (VR2 ${ }^{*} \rightarrow \mathrm{VR}^{*}$ ) [Points to graph]. (VR3*) So we end up with integral of $F$ times $d t$. [Finishes writing: $P=$ $\left.\int F d T\right](\mathrm{VR} 3 *)$

Notice the gestures that connect the product idea to the thin rectangle, the pronoun references to previous ideas ("those quantities," "this really small change in time"), and the use of at least some experiential language to connect with a mental model of the physical situation ("impact").

At some points in her presentations, Amber connected a single aspect of the problem using three different representations. In the passage quoted, Amber connects three representations indirectly, by tying a verbal description and a symbolic expression to the same graph. She states that "once the impact ends [the force is] gonna start to decrease," indicating a force versus time graph. She later attaches the symbolic expression " $d t$ " to the same graph. This chain of representations might be represented as a time-ordered sequence: verbal $\rightarrow$ graphical $\rightarrow$ symbolic. However, Amber's first connection (increasing and then decreasing force) pertains to a different aspect of the graph than Amber's second connection (a small amount of time). This is a nuance that the time-ordered sequence cannot portray. At this point in the sequence of lessons, we transitioned to considering spatial integrals.

*4.b. Amber thought about how she would communicate about the quality of dots on a line by which they could be closer together or farther apart. She came up with the idea of counting the distance between dots, as well as the number density. She learned the macroscopic equation $N=\rho_{N} L$, with $\rho_{N}$ representing the number density, which was to be a model for mass densities later. Density is crucial in many spatial integrals, and we wanted to consolidate the idea.

4.c. We discussed a hanging slinky, in which the rings are farther apart at the top and closer together at the bottom. The number density of the rings can be measured, but the macroscopic definition $\rho_{N}=N / L$ is meaningful only when the length $L$ is larger than the distance between rings and smaller than the length of the slinky.

\section{E. Lesson Five}

5.a. In a spatial integral, infinitesimal quantities such as $d x$ and $d m$ are "amounts" rather than "change quantities." That is, $d m$ is best thought of as a small amount of mass, rather than a small change in a "mass variable" $m$ (whatever that would mean). This distinction is illustrated by the fact that $d x$ cannot be negative in a spatial context, but can be negative in a kinematics context. We gave several examples to talk about these issues.

5.b. Similar to (3.a), Amber thought about the physical quantities that could be associated with the slinky. 


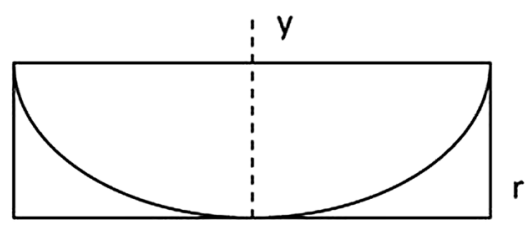

FIG. 3. A diagram of the trough. The water in the trough has a parabolic shape, $y=a r^{2}$, and in the third dimension (into or out of the page), the trough has a constant depth $D=0.6 \mathrm{~cm}$. The trough is sitting on a turntable, which is rotating.

5.c. Amber observed a rotating trough of water on a turntable, in which the rotation led the water to push toward the edges of the container, forming the shape of a parabola. A drawing of the water in the trough is shown in Fig. 3, as well as in Amber's sketch, Fig. 4.

We helped Amber to deduce the moment of inertia of this system. As mentioned in lesson 3.a, Amber needed to know which physical quantities might be relevant in the situation, since no variables, apart from moment of inertia, were specified in the problem. She recognized on her own that density and volume were important, but needed more help setting up the spatial coordinates, $y \propto x^{2}$. After solving the problem, Amber gave a presentation at the blackboard about the layers involved in this process (see Fig. 4). This time, we did not assist her by giving a list of the representations she should use.

In her presentation for lesson five, Amber made the connections shown in Table III.

Amber was able to talk about the infinitesimal equation for volume in a confident way, combining verbal description, equations, and diagrams. In the following passage, she has drawn a diagram of the trough, as shown in Fig. 4.

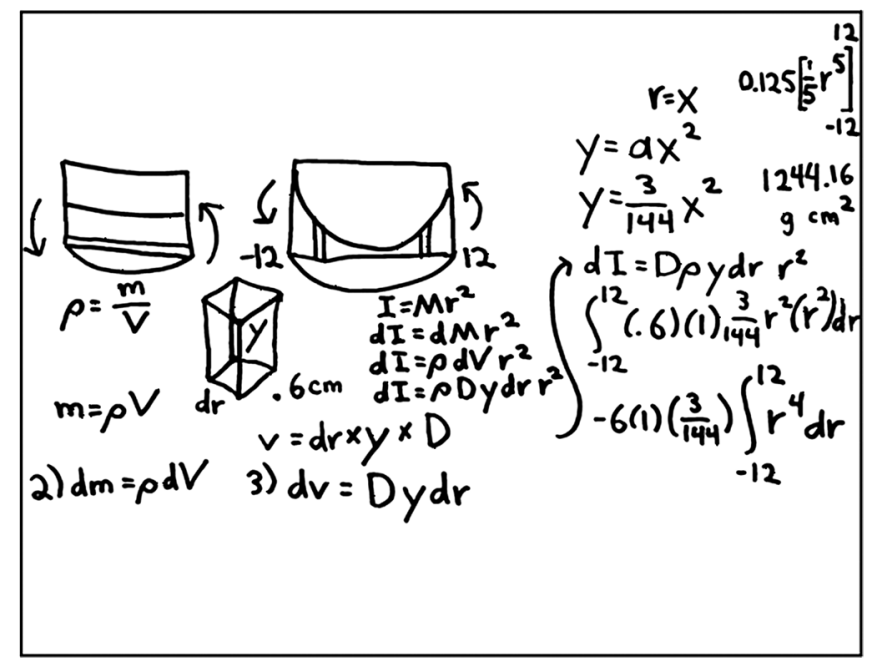

FIG. 4. Sketch of the blackboard after Amber's lesson 5 presentation.
Amber: Okay, so, after you find that, you need to find some way to get the mass of the small section. And so to do that you have, you know the equation "density equals mass over volume" [writes $\rho=m / V]$. So mass equals density times volume [writes $m=\rho V]$. So the density of the water is gonna be the same throughout, but the volume will change based on where you are looking at on the graph, because the radius is gonna change depending on how far out you go. [Indicates points $1 / 3,2 / 3$, and $3 / 3$ of the way out from the center on the picture of the trough of water.] So if you take a small section of this [draws two very close together vertical lines, this time on the left half of the trough] to find density you have sort of [draws a tall $3 D$ rectangular box] like a small cyl... like a small cubic rectangle.

Amber went on to assign values or variables to each of the three dimensions of the "cubic rectangle," constructing an infinitesimal equation (" $d V=y D d r$ ") for the volume.

\section{F. Lesson Six}

6.a.* One way to test knowledge about integration would be to assign a student to set up integrals in a novel context. We attempted this only in lesson six, using a previously tested set of problems. We assigned a set of problems that we had used for another research project [13], concerning work and spatial integrals. These problems scaffolded the construction of work, mass, and moment of inertia integrals, and then we asked the student to set up center-ofmass and torque integrals.

We found that Amber was able to set up a center-of-mass integral, which she had never been asked to consider before. In our previous work, only a small number of students could set up this integral, and most of those who did required multiple hints or else showed signs that they may have learned about this integral before. Amber did not require any hints during the solution process itself.

The center-of-mass problem was a post-test, which came with practice problems about work and moment of inertia that Amber (and the past students) solved in preparation for the post-test. Amber asked more insightful questions about the practice problems than the students in the previous study, requiring and receiving more help. We believe that Amber was able to ask the right questions because of our lessons, but also because there were no other students competing for the researcher's attention. Certainly, Amber's questions were thoughtful and to the point, indicating that she was thinking in the right direction already. For instance, after learning that the mass is $\int \rho d x$ and moment of inertia $\int \rho x^{2} d x$, she wanted to know why moment of inertia could not be found by $\left(\int \rho d x\right) R^{2}$. Although her intuition was wrong in this case, this kind of "why" question was rare or absent among students who had not participated in our series of lessons. 


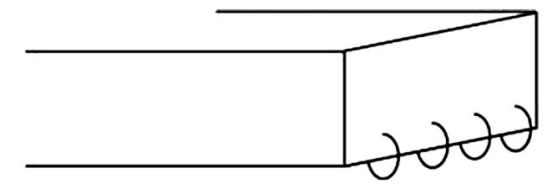

FIG. 5. Hinged door and channel for lesson 7.

6.b. We briefly went over the proof, using infinitesimals, that kinetic energy is $\frac{1}{2} m v^{2}$, by integrating the force over $d x$.

\section{G. Lesson Seven}

7.a. We discussed a problem about the hydrostatic force and torque exerted by a body of water on a hinged door. The door closes a channel, as shown in Fig. 5, so that the water exerts a torque about the hinge.

Amber was asked to recall the derivations of the infinitesimal equations for moment of inertia and torque. The instructor helped Amber to work out the solution to the problem and then gave a presentation about the force part of the problem. Then Amber presented the (more difficult) torque portion.

This lesson differed from all of the others in that Amber watched the researcher give a presentation prior to Amber's own presentation. It is instructive to compare Amber's presentation to the researcher's presentation. Both the researcher and Amber used the product layer (VR2*) in the equation representation, but did not explicitly mention summation. Both drew geometric quantities on the diagram, such as width and height segments, to represent the infinitesimal area $d A=w d x$, although Amber wrote only a lowercase " $a$ " to represent this area, with no " $d$," whereas the researcher wrote " $d A$." The researcher also related pressure and force to the diagram, by drawing circles to represent small regions on which the force was acting, gesturing vertically to indicate that the pressure varied with height, and gesturing horizontally to say that it did not vary with width. Amber did not spontaneously relate pressure or force to the diagram. When we asked Amber the right question, after her presentation was over, she did represent the varying pressure by drawing it on the diagram. This happened when we asked her why the dam should be cut into horizontal strips and not vertical strips, and she responded, "So the reason you want to divide it up horizontally, if you have a rectangle like this [vertical rectangle], it's gonna have a different pressure at each height [draws series of dashes to indicate the varying pressure], so it will not work." This incident exemplifies the kind of information we can gain by asking questions after a presentation.

\section{RESULTS AND DISCUSSION}

As evidenced by her performance in presentation and storytelling problems, Amber's proficiency with layers and representations was substantial in the later lessons. Tables II and III indicate that Amber connected different representations, weaving them together into a coherent story. In lesson five, she connected the diagram, equation, and experiential representations into a single unified argument, and she did this without any hints about the correct representations to use.

Another way to assess Amber's skill is to notice the aspects of her presentations that were most innovative and least like the representations she had recently learned about. For instance, her depiction of a narrow box-shaped volume element in lesson five was unlike anything that she had seen in these lessons; she may have transferred her knowledge from calculus to the physics context. In lesson four, Amber drew graphs not only of the integrand $F(t)$, but also of the integral function (VR4*) $p(t)$, which she had not previously been asked to do. She also explicitly wrote down quantitative comparisons between physical quantities as time progressed, stating that at successive times 1 and 2, $F_{1}<F_{2}$ and $v_{1}>v_{2}$. She had not been instructed to write symbolic comparisons in connection with the table representation.

Amber's behavior in lesson six also provided evidence of improved understanding. She approached a quantitative integration problem in a different way than other students we had worked with, asking sense-making questions during the scaffolding process and then solving the center-of-mass problem without too much difficulty. It is difficult to compare her behavior with that of past students, since her lessons were one on one and the other students worked in groups. However, her ability to ask insightful questions and to get the right answer was a positive improvement relative to past students working on these problems.

Both Amber's essay in lesson two and her presentation in lesson four navigate the layers of integration, but they achieve this goal in slightly different ways. In lesson two, Amber imagines a limit being taken after finitely many quantities are summed. In lesson four, she talks about a sum of small quantities and does not mention a limit. The former might be called the "limit of the sum" perspective, or $\left(\mathrm{VR} 1 \rightarrow \mathrm{VR} 2 \rightarrow \mathrm{VR} 3 \rightarrow \mathrm{VR}^{*}\right)$, and the latter is the "sum of small quantities" perspective, or (VR1* $\rightarrow$ VR2 $\rightarrow$ VR3*). Our framework permits either sequence, whereas other authors privilege the "limit of the sum" perspective. We feel that the "sum of small quantities" perspective is acceptable and sufficient in a physics class, if not in a math class, and deserves a place in our framework.

At the end of the sequence of lessons, we asked Amber which exercises had been most helpful to her. She cited the initial debate problem about " $d x=v d t$." She enjoyed McDermott's representations of motion tutorial, as well as the one-hour review lecture in lesson three. The presentation in lesson four was confusing for her since it was unclear to her what we wanted her to do (although in our opinion she did a good job of interpreting our intent nevertheless.) The slinky exercise in lessons 4.b and 4.c helped her to make sense of the idea of density. The distinction between the amount " $d x$ " and the change " $d x$ " was 
beneficial, as well as an example we gave about finding the moment of inertia of a rod rotating about an axis parallel to itself. A torque debate problem in lesson six was helpful even though it was difficult and she did not find the right answer. Finally, the hydrostatic dam problem in lesson seven was useful in consolidating ideas about torque, pressure, and some concepts from her calculus course.

\section{CONCLUSIONS AND FUTURE WORK}

In this paper, we have discussed the significance of layers and representations in a sequence of lessons about setting up integrals. A student cannot be said to fully understand integration in a physics context unless she can make use of several layers, such as the quantity, product, and sum layers. Building an integral requires being aware of the relevant physical quantities, both macroscopic and infinitesimal, constructing the appropriate product from these quantities, and adding a sequence of products to arrive at a sum or integral. However, awareness of these layers is not enough. A student needs to know what is being added up and why, not only the layer structure of the process. Thus, the student must be able to relate each layer of the integral to several different representations, including graphical, verbal, equation, and concrete physical representations.

Our lessons helped an introductory mechanics student, "Amber," to become aware of these layers and representations. We built on Amber's experience with the physical world, while avoiding premature connections with her calculus knowledge. In order to test Amber's understanding, we asked her to make presentations about integration at the blackboard. We were able to judge her presentations by noticing when she linked multiple representations and layers in a fluid way.

One might imagine assessing knowledge about integration using quantitative problems, perhaps by asking a student to set up an integral in an unfamiliar situation. However, we expect that most introductory mechanics students will require some help to set up unfamiliar integrals, even if they have a fair amount of experience with multiple layers and representations. A more appropriate assessment, which can reveal degrees of understanding, is to ask the student to make a presentation about a more familiar topic. In this situation, students have a chance to display some degree of understanding, even if they cannot solve unfamiliar integral problems.

Although oral presentations are an informative assessment tool, one might ask whether they are practical in the context of a large lecture course. In a large course, instructors do not have time to observe presentations from every student, handle the logistics of a presentation schedule, and grade every presentation. We can think of a few solutions to these challenges. First, the scheduling logistics become easier if students give presentations not to a live audience, but to a recording device. For instance, a "Smartpen" audio pen recorder is capable of storing both speech and writing. Second, teaching assistants or learning assistants could be trained to observe and grade presentations. Third, undergraduates themselves could grade their own presentations or others' presentations. Fourth, the instructor could gauge the students' collective understanding by viewing presentations from a small sample of individuals. We have not established the viability of these schemes, but we hope to investigate these possibilities in the future.

Another task for future research would be to perform a controlled study about the effectiveness of this kind of intervention. When designing such a study, one important question is what the control group will be like. Because calculus-based mechanics courses can vary dramatically with respect to their treatment of integration, there is a real dilemma about whether the control group should receive instruction in a traditional curriculum (and if so, whose curriculum?) or a curriculum designed expressly for the purpose of helping students to make presentations about integration. (And if so, designed by whom?). One must also decide whether the control group should experience a traditional teaching method such as lecture, or a nontraditional method. Still, a controlled study could provide quantitative evidence that our intervention played a role in generating students' understanding.

Although Amber's experience should not be taken as typical of all students, her increasing skill with representations illustrates the type of learning that is possible for at least some first-semester calculus students, as well as the value of presentation as an assessment tool. In the future, we hope to test these ideas with a larger body of students enrolled in the same introductory mechanics course.

\section{ACKNOWLEDGMENTS}

The authors would like to thank Dehui $\mathrm{Hu}$, Bashirah Ibrahim, Sytil Murphy, Eleanor Sayre, and Kim Coy for their advice and help. This project is supported by the National Science Foundation under Grant No. 0816207.

\section{APPENDIX}

This Appendix contains a selection of the problems from our seven lessons. Statements marked "note to the reader" did not appear in the original lesson; they are clarifications for the benefit of the readers of this paper.

\section{Lesson 1.a}

Note to the reader-The three students were instructed to discuss and write down their thoughts about these questions, even if they were not certain about their answers.

Question 1: "A malfunctioning speedometer"

**P1. Your iPhone has a "speedometer" app that prints on the screen how fast you are moving, in miles per hour. You are driving with your friends Dan, Claire, and Paul on a long road trip, and comparing the iPhone measurement with the car's actual speedometer. And they are different! One of them says $65 \mathrm{mph}$, and the other says $75 \mathrm{mph}$. You have the 
trip planned out to get to Saint Louis at a certain time, so you'd like to know the speed. But you cannot trust either the car or the app, because they do not agree. What else can you do to help figure out how fast the car is going?

Question 2: "A debate"

When the speedometer malfunctions, you tell your friends: "Why don't we do this using physics, using the equation $x=v t$ ?" Dan wants to know, "But what numbers should we use for $x, v$, and $t$ ?" Your friends have several different ideas about how to do this. Carefully read and discuss what each of them has to say. They may be partly right and partly wrong.

Here's what Dan has to say:

The place where $x$ starts has to be the starting point of our trip. At the beginning of the trip, the car's main odometer read 270 miles. (The car is very new.) Now it says 412 miles. So we have gone $412-270=142$ miles, and that is $x$, our change in position relative to the start.

In physics, everything is relative. What "velocity" is supposed to mean is "velocity relative to the start of the trip." So $v$ is our change in velocity relative to the start. I guess the trip really "started" when we got onto the highway, so $v$ means the difference between our velocity now, and our velocity at that time, $v_{\text {now }}-v_{\text {start }}$.

And $t$ is our change in time relative to the start. Since we started 2 hours ago, that is the time, $t$, in the equation.

Here's what Claire has to say:

I think the $x$ should be a small change in position. So we should pick two points: one now, and one a short ways ahead. Maybe in 1 mile. Then $x$ is one mile.

The equation is about small changes. So $v$ should be a small change in velocity. $v$ will be how much our velocity changed between the two points. If we were going $60 \mathrm{mph}$, and later we are going $61 \mathrm{mph}$, then $v$ will be that small difference, $1 \mathrm{mph}$.

And it seems like $t$ should be a small change in time, because like I said the equation is about small changes. So $t$ will be how much the time changes between the two points. If we pick a point 0.1 hours ahead, then $t$ will be the small difference, 0.1 hours.

Here's what Paul has to say:

Obviously, the $x$ is the odometer reading of our car, which is our position at this point. The main odometer reads 412 miles, which is our position, $x$.

Clearly, the $v$ is our velocity at this point, in miles per hour. If the speedometer were not broken, then whatever it says would be $v$. It's probably either $65 \mathrm{mph}$ or $75 \mathrm{mph}$, depending on whether you trust the speedometer or the iPhone.
And anyone can see that $t$ is our time at this point, in hours. Since it is $12: 30 \mathrm{pm}$, and 30 minutes is half an hour, the answer is that $t=12.5$ hours.

** P2.a. What parts are right and what parts are wrong, and how do you know?

** P2.b. In your own words, what pattern connects the three different things that Dan says? How about the three things Claire says? How about Paul?

** P2.c. How would you convince your three friends about the right answer?

Question 3: "A word problem" [we skipped this question, which asked students to construct a word problem]

Question 4: "Some symbols"

Let's find some symbols to talk in Paul's way, Dan's way, and Claire's way. Of course we could write $v_{\text {Paul }}$, $v_{\text {Dan }}, v_{\text {Claire }}$ to make clear whose velocity we are talking about. But let's find a quicker way of writing it. Suppose each of them wants to talk about the velocity, $v$. Then:

Dan's symbol is $\Delta$, so " $\Delta v$." This triangle is really a letter of the Greek alphabet, an uppercase "Delta." (Lowercase "delta" is written $\delta$, you will not see it in this lesson.)

Claire's symbol is $d$, so " $d v$."

Paul's symbol is no special symbol, just " $v$."

Remember, Claire likes small changes, or you might say "differences." That's why her letter is a small " $d$," for "differences." Dan likes big changes. That's why his letter is big Delta for big "Differences." So we say that

Dan is talking about $\Delta x, \Delta v$, and $\Delta t$.

Claire is talking about $d x, d v$, and $d t$.

Paul is talking about $x, v$, and $t$.

P4. Rewrite the equation " $x=v t$ " in the correct form used to find the velocity of your car, from question 2 .

This is the "equation for infinitesimal motion." It's important that you remember what you did in question 2 to help you get to this equation. Do not just memorize the equation itself.

Question 5: [we skipped this question, which was about average velocity]

\section{Lesson 2.a, 2.b}

Sequence diagram for the motion of a car

(Figure 6 was inserted at this point in lesson 2.)

1. Draw a picture of the motion of the car. Describe in words what it might be doing?

2. Write down an equation that you could use to approximate:

(a) The horizontal displacement from frame \#1 to \#6? $\left(\Delta x=x_{6}-x_{1}\right)$

(b) The vertical displacement from frame \#1 to \#6? $\left(\Delta y=y_{6}-y_{1}\right)$

3. These people have different opinions about the equation for (2.a). 


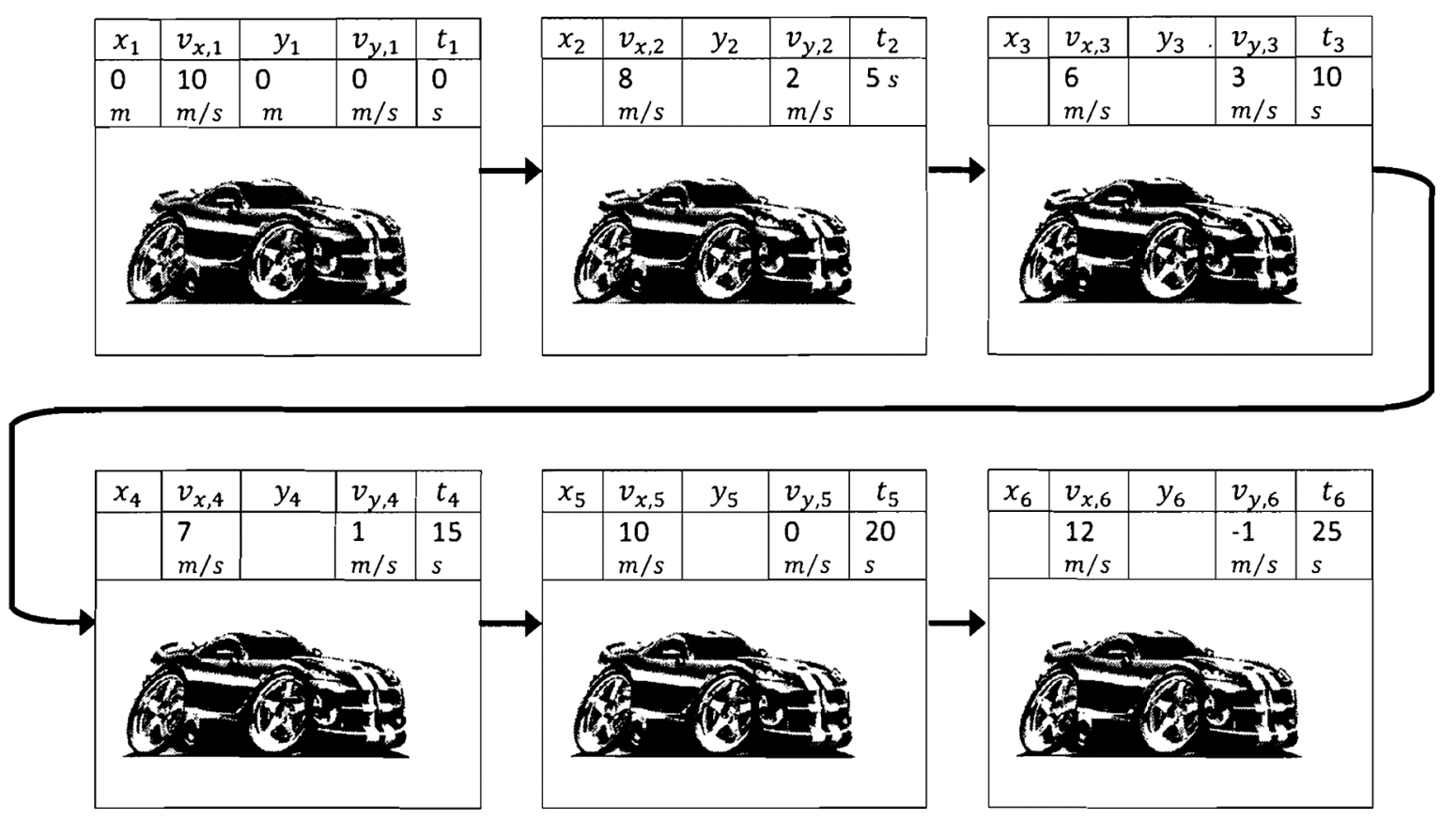

FIG. 6. "Sequence diagram" of a car used in lesson 2.

Which ones, if any, are right? (like " $2+2=4$ ")

Which ones, if any, are wrong? (like " $2+2=5$ ")

Which ones, if any, are meaningless? (like " $2+-* 2=5$ ")

Calculate the first term of each, just to show how it would be done. (Of course, if the equation is meaningless, this may not be possible.)

** How would you convince these people that their equations are wrong or meaningless?

(a) Charles: "The equation is $\Delta x \approx v_{\text {total }} t_{\text {total }}$. This is a quick way of summarizing the whole series of numbers."

(b) Sandy: "The equation is $\Delta x \approx v_{1, x} d t_{1}+v_{2, x} d t_{2}+$ $v_{3, x} d t_{3}+v_{4, x} d t_{4}+v_{5, x} d t_{5}$. We are adding up the little displacements from the different small amounts of time."

(c) Jackie: "The equation is $\Delta x \approx t_{1} d v_{1, x}+t_{2} d v_{2, x}+$ $t_{3} d v_{3, x}+t_{4} d v_{4, x}+t_{5} d v_{5, x}$. This is basically the same as Sandy's equation, except it emphasizes the changing velocity."

(d) Matt: "The equation is $\Delta x \approx v_{1} d t_{1}+v_{2} d t_{2}+$ $v_{3} d t_{3}+v_{4} d t_{4}+v_{5} d t_{5}$. So, " $v_{1}$ " is not in the sequence diagram, but what I mean is the speed in frame 1, which is the magnitude of the velocity vector. That's $v_{1} \approx \sqrt{v_{1, x}^{2}+v_{1, y}^{2}}$. You have to count the whole speed, not just a component."

\section{Lesson 2.d}

Tell the story

Question: In the last two labs, we have done a lot. We started with an equation $x=v t$ (a week ago), and we ended up finding displacement as an integral or an area under a curve. Write about how this happened. What logical steps did we follow to make this connection? How did we figure it out? How would you explain to a classmate who had not taken these labs, and wants to know why the displacement is $\Delta x=\int v d t$ ?

Note to the reader-The image of the car was obtained from Ref. [17].

\section{Lesson 3.a}

(1) List some physical variables that might be of interest during the motion of the glider. A physical variable is any kind of number or variable that physicists might care about, that is related to this experiment.

(2) Perform the experiment, and fill in the values of the variables into a table. Then, write four adjacent rows of the table into a sequence diagram.

A discussion about infinitesimal equations.-This is an infinitesimal equation: $d x=v d t$.

Write down some things we have learned about it so far. Can you say anything about: The difference between " $d x$ ", " $\Delta x$ ", and " $x$ "?

How " $d x=v d t$ " is related to integrals?

How " $d x=v d t$ " is related to sequence diagrams?

" $x=v t$ " was an equation that we turned into the infinitesimal equation, " $d x=v d t$." Do you know any other equations that we could turn into infinitesimal equations? What integrals could we make out of them? Write some ideas here. 


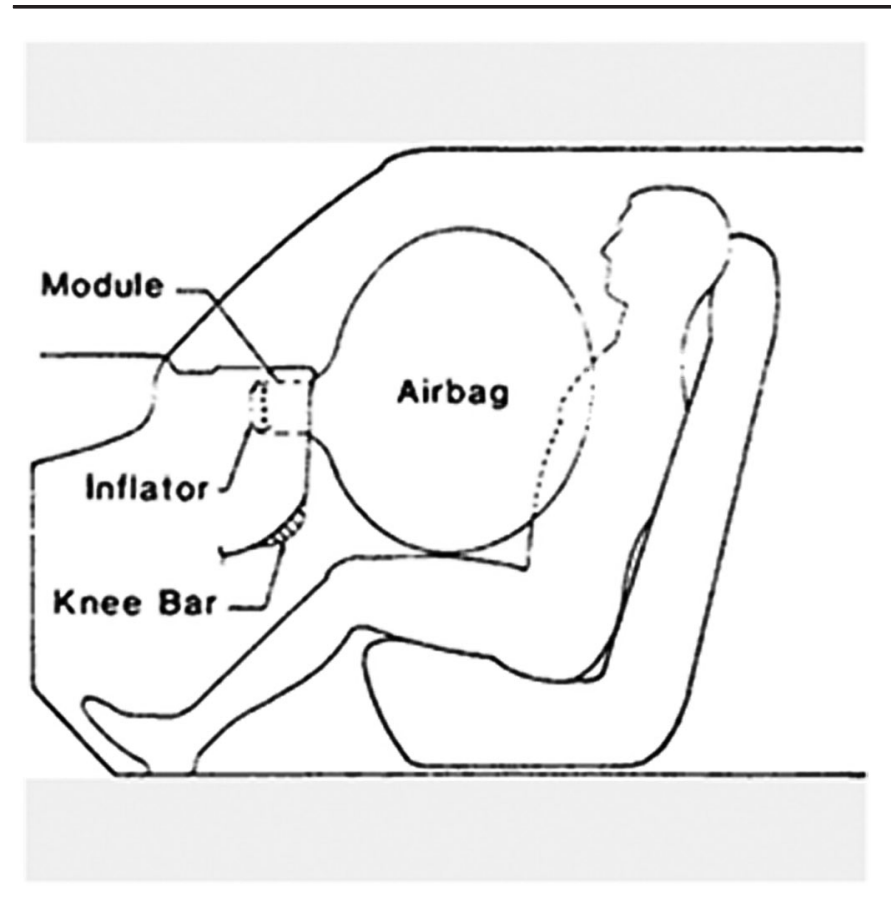

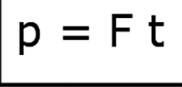

FIG. 8. Equation used in lesson 4.
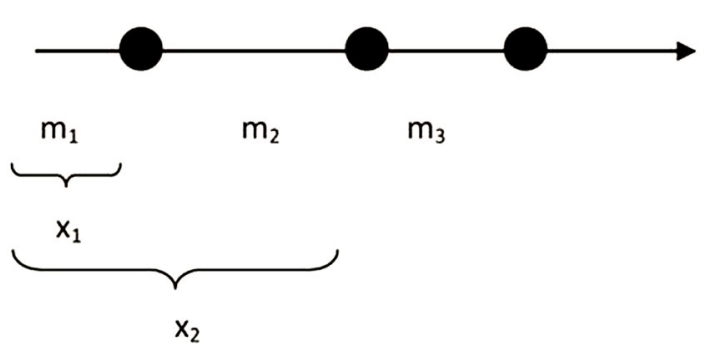

FIG. 9. Discrete center-of-mass diagram used in lesson 6.

FIG. 10. Diagram of a rod used in lesson 6.

(like Paul's idea in lesson 1), infinitesimal quantities (like Claire's idea in lesson 1), and interval quantities (like Dan's idea in lesson 1).

Another note to the reader.-The image of the airbag was obtained from Ref. [18].

\section{Lesson 6.a}

Center-of-mass. For a system of $n$ point particles located on the $x$ axis at positions $x_{1}, x_{2}, x_{3} \ldots$, the center-of-mass is $X_{\mathrm{cm}}=\frac{m_{1} x_{1}+m_{2} x_{2}+m_{3} x_{3}+\ldots}{M}$, where $M=m_{1}+m_{2}+m_{3}+$ $\cdots$ is the total mass.

(Figure 9 was inserted at this point in lesson 6.)

Now, imagine a rod with a changing linear density $\rho(x)=$ $x^{3}+2 x^{2}+2$, where $x$ is in meters and $\rho(x)$ is in $\mathrm{kg} / \mathrm{m}$. The length of the rod is 2 meters. The linear density $\rho$ of a rod is defined as its mass divided by its length. If the linear density $\rho(x)$ is changing as a function of $x$, it means that a small segment of the rod near point $x$ has density $\rho(x)$.

(Figure 10 was inserted at this point in lesson 6.)

Find the center-of-mass of the rod.
[1] D. C. Meredith and K. A. Marrongelle, How students use mathematical resources in an electrostatics context, Am. J. Phys. 76, 570 (2008).

[2] D. Nguyen and N. S. Rebello, Students' difficulties with integration in electricity, Phys. Rev. ST Phys. Educ. Res. 7, 010113 (2011).
[3] M. Zandieh, A theoretical framework for analyzing student understanding of the concept of derivative, in Research in Collegiate Mathematics Education IV, edited by E. Dubinsky, A. H. Schoenfeld, and J. Kaput (American Mathematical Society, Providence, 2000), p. 103. 
[4] P.W. Thompson and J. Silverman, The concept of accumulation in calculus, in Making the Connection: Research and Teaching in Undergraduate Mathematics, edited by M. P. Carlson and C. Rasmussen (Mathematical Association of America, Washington, DC, 2008), p. 43.

[5] V. Sealy, Ph.D. thesis, Arizona State University, 2008.

[6] A. Sfard, On the dual nature of mathematical conceptions: Reflections on processes and objects as different sides of the same coin, Educ. Stud. Math. 22, 1 (1991).

[7] J. Hiebert and P. Lefevre, Conceptual and procedural knowledge in mathematics: An introductory analysis, in Conceptual and Procedural Knowledge: The Case of Mathematics, edited by J. Hiebert (Erlbaum, Hillsdale, NJ, 1986).

[8] R. Lesh, and M. Landau, Acquisition of Mathematics Concepts and Processes (Academic, New York, 1983).

[9] R. R. Skemp, The Psychology of Learning Mathematics (Penguin Books, Harmondsworth, England, 1971).

[10] Munirah Ghazali et al., Dominant representation in the understanding of basic integrals among post secondary students, in Proceedings of the Eighth International Conference on Reform, Revolution and Paradigm Shifts in Mathematics Education, Johor Bahru, Malaysia, 2005, edited by A. Rogerson, http://math.unipa.it/ grim/ 21_project/21_malasya_Ghazal97-100_05.pdf.

[11] P. N. Johnson-Laird, Mental Models: Towards a Cognitive Science of Language, Inference, and Consciousness (Harvard University Press, Cambridge, 1983).

[12] D. C. Phillips, The good, the bad, and the ugly: the many faces of constructivism, Educ. Researcher 24, 5 (1995) [http://www.jstor.org/stable/1177059].

[13] D. Hu, J. Von Korff, and S. Rebello (unpublished).

[14] C. Hieggelke, D. Maloney, and S. Kanim, Newtonian Tasks Inspired by Physics Education Research: nTIPERs (Pearson Education, San Francisco, 2012).

[15] L.C. McDermott and P.S. Shaffer, Tutorials in Introductory Physics (Prentice Hall, New Jersey, 1998).

[16] D. L. Schwartz and J.D. Bransford, A time for telling, Cogn. Instr. 16, 475 (1998).

[17] http://www.carbodydesign.com/tutorial/2230/drawing-acartoon-car/

[18] http://www.procarcare.com/icarumba/resourcecenter/ encyclopedia/icar_resourcecenter_encyclopedia_safety1.asp 